\title{
INVARIANT PROBABILITIES FOR FELLER-MARKOV CHAINS ${ }^{1}$

\author{
ONÉSIMO HERNÁNDEZ-LERMA \\ CIN VESTA V-IPN \\ Departamento de Matemáticas
} \\ A. Postal 14-740, 07000 México D.F., México \\ E-mail: ohernand@math.cinvestav.mx
}

\author{
JEAN B. LASSERRE \\ $L A A S-C N R S$ \\ 7 Av. du Colonel Roche \\ 31077 Toulouse Cedex, France \\ E-mail: lasserre@laas.fr
}

(Received April, 1995; Revised August, 1995)

\begin{abstract}
We give necessary and sufficient conditions for the existence of invariant probability measures for Markov chains that satisfy the Feller property.

Key words: Markov Chains, Feller Property, Invariant Measures.

AMS (MOS) subject classifications: 60J05, 93E15.
\end{abstract}

\section{Introduction}

The existence of invariant probabilities for Markov chains is an important issue for studying the long-term behavior of the chains and also for analyzing Markov control processes under the long-run expected average cost criterion. Inspired by the latter control problems, we present in this paper, two necessary and sufficient conditions for the existence of invariant probabilities for Markov chains that satisfy the Feller property. Our study extends previous results using stronger assumptions, such as the strong Feller property in Beneš [1], nondegeneracy assumptions (see condition (2) in Beneš [2]), and a uniform countable-additivity hypothesis in Liu and Susko [8]. As can be seen in the references, it is also worth noting that there are many reported results providing (only) sufficient conditions for the existence of invariant measures; in contrast however, our conditions are also necessary. 3.

The setting for this paper is specified in Section 2, and our main result is presented in Section

\footnotetext{
${ }^{1}$ This work is part of a research project jointly sponsored by CONACYT (México) and CNRS (France). The work of the first author was also partially supported by CONACYT grant 1332-
} E9206. 


\section{Notation and Definitions}

Let $X$ be a $\sigma$-compact metric space, and let $\left\{x_{t}, t=0,1, \ldots\right\}$ be an $X$-valued Markov chain with time-homogeneous kernel $P$, i.e.,

$$
P(B \mid x)=\operatorname{Prob}\left(x_{t+1} \in B \mid x_{t}=x\right) \forall t=0,1, \ldots, x \in X, B \in \mathfrak{B}(X),
$$

where $\mathfrak{B}(X)$ denotes the Borel $\sigma$-algebra of $X$. A probability measure (p.m.) $\mu$ on $\mathscr{B}(X)$ is said to be invariant for $P$ if

$$
\mu(B)=\int_{X} P(B \mid x) \mu(d x) \quad \forall B \in \mathscr{B}(X) .
$$

Here, we give necessary and sufficient conditions for the existence of invariant p.m.'s when $P$ satisfies the Feller property:

$$
x \rightarrow \int u(y) P(d y \mid x) \text { is in } C(X) \text { whenever } u \in C(X),
$$

where $C(X)$ denotes the space of all bounded and continuous functions on $X$. Our conditions use a moment function, defined as follows.

Definition: A nonnegative Borel-measurable function $v$ on $X$ is said to be a moment if, as $n \rightarrow \infty, \inf \left\{v(x) \mid x \notin K_{n}\right\} \uparrow \infty$ for some sequence of compact sets $K_{n} \uparrow X$.

Moment functions have been used by several authors to study the existence of invariant measures for Markov processes (e.g., see Beneš [1, 2], Hernández-Lerma [6], Liu and Susko [8], and Meyn and Tweedie [9]). The key feature used in these studies is the following (easily proved) fact.

Lemma: Let $M$ be a family of p.m.'s on $X$. If there exists a moment $v$ on $X$ such that sup $_{\mu \in M} \int v d \mu<\infty$, then $M$ is tight, i.e., for every positive $\epsilon$ there exists a compact set $K$ such that $\mu(K)>1-\epsilon$ for all $\mu \in M$.

Therefore by Prohorov's Theorem [3,9], the family $M$ in the lemma is relatively compact, i.e., every sequence in $M$ contains a weakly convergent subsequence.

Our theorem below (see Section 3) extends a result by Benes [2] where our conditions ( $a$ ) and (b) are new and, most importantly, we do not require Benes' "nondegeneracy" condition, according to which

$$
\lim _{x \rightarrow \infty} P^{t}(K \mid x)=0 \text { for } t=1,2, \ldots, K \text { compact, }
$$

with $P^{t}(\cdot \mid x)$ being the $t$-step transition probability given the initial state $x_{0}=x$. This condition excludes important classes of ergodic Markov chains, such as those that have a "minorant"; see e.g., Dynkin and Yushkevich [5], or condition R1 in Hernández-Lerma et al. [7]. See also Remarks 2 and 3 (Section 3 ) for additional comments on related results.

\section{The Theorem}

If $\nu$ is a p.m. on $X, E_{\nu}(\cdot)$ stands for the expectation given the "initial distribution" $\nu$.

Theorem: If $P$ satisfies the Feller property, then the following conditions $(a),(b)$, and $(c)$ are equivalent: 
(a) There exists a p.m. $\nu$ and a moment $v$ such that

$$
\limsup _{n \rightarrow \infty} J_{n}(\nu)<\infty
$$

where $J_{n}(\nu):=n^{-1} E_{\nu}\left[\sum_{t=0}^{n-1} v\left(x_{t}\right)\right]$;

(b) There exists a p.m. $\nu$ and a moment $v$ such that

$$
\underset{\alpha \uparrow 1}{\lim \sup } V_{\alpha}(\nu)<\infty,
$$

where $V_{\alpha}(\nu):=(1-\alpha) E_{\nu}\left[\sum_{t=0}^{\infty} \alpha^{t} v\left(x_{t}\right)\right]$;

(c) There exists an invariant probability for $P$.

Proof: We will show that $(a) \Rightarrow(b) \Rightarrow(c) \Rightarrow(a)$.

(a) implies (b): This follows from a well-known Abelian theorem (e.g., see Sznajder and Filar [11], Theorem 2.2), which states that

$$
\limsup _{\alpha \uparrow 1} V_{\alpha}(\nu) \leq \limsup _{n \rightarrow \infty} J_{n}(\nu) .
$$

(Since a direct proof that $(a)$ implies $(c)$ is surprisingly simple, it will also be included here; see Remark 1 below.)

(b) implies $(c)$ : Suppose that $(b)$ holds and for each $\alpha \in(0,1)$, let $\mu_{\alpha}$ be the probability measure on $X$ defined as

$$
\mu_{\alpha}(B):=(1-\alpha) \sum_{t=0}^{\infty} \alpha^{t} \int_{X} P^{t}(B \mid z) \nu(d z), \quad B \in \mathscr{B}(X) .
$$

Then we may write $V_{\alpha}(\nu)$ as $V_{\alpha}(\nu)=\int v d \mu_{\alpha}$. Let $\left\{\alpha_{n}\right\}$ be a sequence in $(0,1)$ such that $\alpha_{n} \uparrow 1$ and, by $(b)$,

$$
\limsup _{\alpha \uparrow 1} V_{\alpha}(\nu)=\lim _{n \rightarrow \infty} V_{\alpha_{n}}(\nu)=\lim _{n \rightarrow \infty} \int v d \mu_{\alpha_{n}}<\infty
$$

By the lemma in Section 2, $\left\{\mu_{\alpha_{n}}\right\}$ is tight and therefore, by Prohorov's Theorem, $\left\{\mu_{\alpha_{n}}\right\}$ contains a weakly convergent subsequence, which we denote by $\left\{\mu_{\alpha_{n}}\right\}$ again; that is, there exists a probability measure $\mu$ on $X$ such that

$$
\lim _{n} \int u d \mu_{\alpha_{n}}=\int u d \mu \forall u \in C(X) .
$$

We claim that $\mu$ is invariant for $P$.

To see this, first note that by the Markov property, we may write $\mu_{\alpha}$ as

$$
\mu_{\alpha}(B)=(1-\alpha) \nu(B)+\alpha \int P(B \mid x) \mu_{\alpha}(d x) \quad \forall \alpha \in(0,1), \quad B \in \mathscr{B}(X) .
$$

Hence, for any $u \in C(X)$,

$$
\int u d \mu_{\alpha}=(1-\alpha) \int u(x) \nu(d x)+\alpha \iint u(y) P(d y \mid x) \mu_{\alpha}(d x),
$$


and furthermore, note that by the Feller property (1), $\int u(y) P(d y \mid \cdot)$ is in $C(X)$. Thus, replacing $\alpha$ by $\alpha_{n}$ and letting $n \rightarrow \infty$, we obtain

$$
\int u d \mu=\iint u(y) P(d y \mid x) \mu(d x) .
$$

Finally, since $u \in C(X)$ was arbitrary, we conclude from (2) that $\mu$ is invariant for $P$.

(c) implies (a): Let $\nu$ be an invariant probability for $P$, and let $\left\{K_{n}\right\}$ be an increasing sequence of compact sets such that $K_{n} \uparrow X$ and $\nu\left(K_{n+1}-K_{n}\right)<1 / n^{3}, n=1,2, \ldots$ (Here we have used the fact that every p.m. on a $\sigma$-compact metric space is tight; see [3], p. 9.) Define a function $v(\cdot):=0$ on $K_{1}$ and $v(x):=n$ for $x \in K_{n+1}-K_{n}, n \geq 1$. Then $v$ is a moment and

$$
\limsup _{n \rightarrow \infty} J_{n}(\nu)=\int v(x) \nu(d x) \leq \sum_{n=1}^{\infty} n^{-2}<\infty \text {. }
$$

Remark 1: We will prove directly that $(a)$ implies $(c)$. Suppose that $(a)$ holds and for every $n=1,2, \ldots$, let $\mu_{n}$ be the probability measure on $X$ defined as

$$
\mu_{n}(B):=n^{-1} \sum_{t=0}^{n-1} \int P^{t}(B \mid z) \nu(d z), \quad B \in \mathscr{B}(X),
$$

so that we may rewrite the condition in $(a)$ as

$$
\limsup _{n \rightarrow \infty} \int v d \mu_{n}<\infty .
$$

Hence, by the lemma in Section $2,\left\{\mu_{n}\right\}$ has a subsequence $\left\{\mu_{n_{i}}\right\}$ which converges weakly to some probability measure $\mu$. We will show that (cf. (2))

$$
\int L u(x) \mu(d x)=0 \quad \forall u \in C(X)
$$

where $L u(x):=\int u(y) P(d y \mid x)-u(x)$, thus showing that $\mu$ is invariant for $P$.

Indeed, for any bounded measurable function $u$ on $X$, the sequence

$$
M_{n}(u):=u\left(x_{n}\right)-\sum_{t=0}^{n-1} L u\left(x_{t}\right), \quad n=1,2, \ldots,
$$

with $M_{0}(u):=u\left(x_{0}\right)$, is a martingale, which implies

$$
E_{\nu}\left[M_{n}(u)\right]=E_{\nu}\left[M_{0}(u)\right] \forall n,
$$

i.e.,

$$
E_{\nu}\left[u\left(x_{n}\right)\right]-n \int L u(x) \mu_{n}(d x)=\int u(x) \nu(d x) .
$$

Finally, let $u$ be in $C(X)$; replace $n$ by $n_{i}$; multiply by $1 / n_{i}$; and then let $i \rightarrow \infty$ to get (3).

Remark 2: In [8], it is shown that

$$
\sup _{t \geq 1} \iint g(y) P^{t}(d y \mid x) \nu(d x)<\infty
$$

for some moment $g$ and initial p.m. $\nu$, is also a necessary and sufficient condition for existence of invariant probabilities provided that the Markov chain satisfies the uniform countable-additivity property 


$$
\lim _{A \downarrow \emptyset} \sup _{x \in K} P(A \mid x)=0
$$

for every compact set $K$ in $x$.

Note that (4) is stronger than our condition (a) and that (5) implies: For every compact set $K \subset X$, the family of p.m.'s $\{P(\cdot \mid x)\}_{x \in K}$ is tight.

Remark 3: It is worth noting that the theorem still holds if we replace "lim sup" by "liminf" in both conditions $(a)$ and $(b)$. Now, $(b) \Rightarrow(a)$ by a well-known Abelian theorem [11]. With similar arguments as in Remark $1,(a) \Rightarrow(c)$. We finally prove $(c) \Rightarrow(b)$ by exhibiting the same moment function $v$ and show that

$$
\liminf _{\alpha \uparrow 1} V_{\alpha}(\nu)=\liminf _{\alpha \uparrow 1}(1-\alpha) E_{\nu} \sum_{t=0}^{\infty} \alpha^{t} v\left(x_{t}\right)=\int v(x) \nu(d x) \leq \sum_{t=0}^{\infty} n^{-2}<\infty .
$$

In conclusion, we mention that the theorem can be extended in the obvious way to continuous-time Markov processes, as in [2]. Conditions for uniqueness and ergodicity of invariant measures can be found, for instance, in $[4,7,10]$ and references therein.

\section{References}

[1] Beneš, V.E., Existence of finite invariant measures for Markov processes, Proc. Amer. Math. Soc. 18 (1967), 1058-1061.

[2] Beneš, V.E., Finite regular invariant measures for Feller processes, J. Appl. Prob. 5 (1968), 203-209.

[3] Billingsley, P., Convergence of Probability Measures, Wiley, New York 1968.

[4] Borovkov, A.A., Conditions for ergodicity of Markov chains which are not associated with Harris irreducibility, Siberian Math. J. 32 (1992), 543-554.

[5] Dynkin, E.B. and Yushkevich, A.A., Controlled Markov Processes, Springer-Verlag, New York 1979.

[6] Hernández-Lerma, O., Lyapunov criteria for stability of differential equations with Markov parameters, Boletin Soc. Mat. Mexicana 24 (1979), 27-48.

[7] Hernańdez-Lerma, O., Montes de Oca, R. and Cavazos-Cadena, R., Recurrence conditions for Markov decision processes with Borel state space: a survey, Ann. Op. Res. 28 (1991), $29-46$.

[8] Liu, J. and Susko, E., On strict stationarity and ergodicity of a nonlinear ARMA model, $J$. Appl. Probab. 29 (1992), 363-373.

[9] Meyn, S.P. and Tweedie, R.L., Markov Chains and Stochastic Stability, Springer-Verlag, London 1993.

[10] Skorokhod, A.V., Topologically recurrent Markov chains: ergodic properties, Theory Prob. Appl. 31 (1986), 563-571.

[11] Sznajder, R. and Filar, J.A., Some comments on a theorem of Hardy and Littlewood, J. Optim. Theory Appl. 75 (1992), 201-208. 


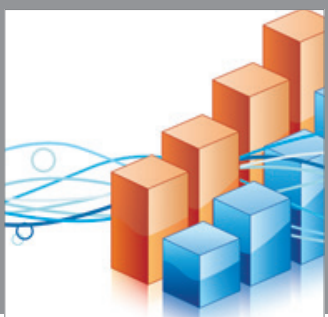

Advances in

Operations Research

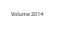

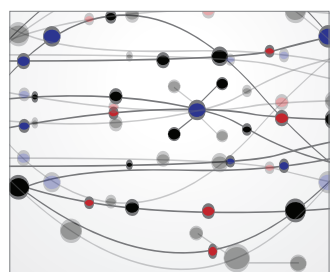

\section{The Scientific} World Journal
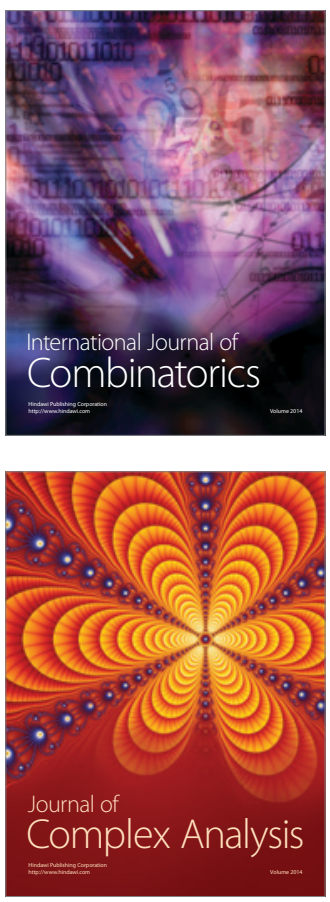

International Journal of

Mathematics and

Mathematical

Sciences
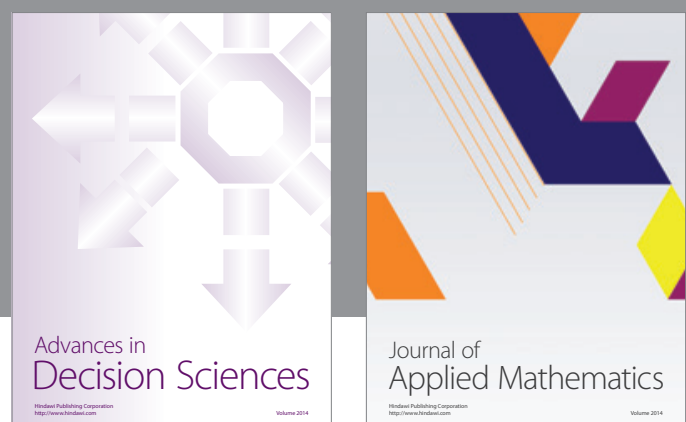

Journal of

Applied Mathematics
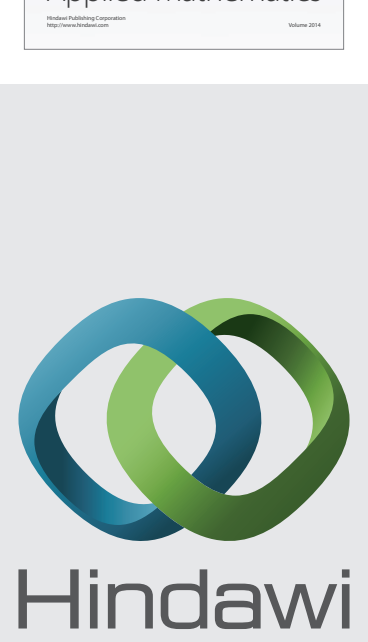

Submit your manuscripts at http://www.hindawi.com
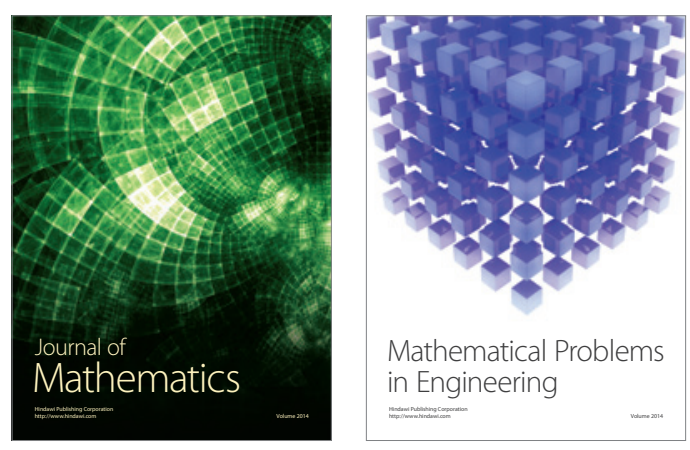

Mathematical Problems in Engineering
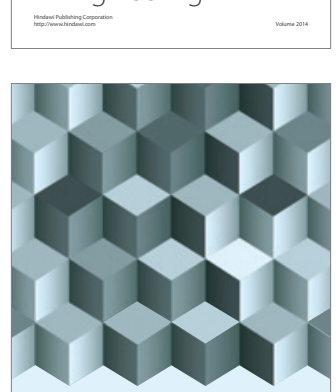

Journal of

Function Spaces
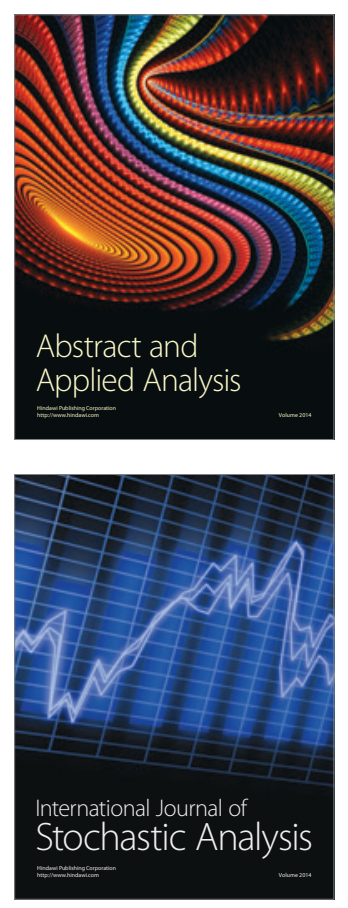

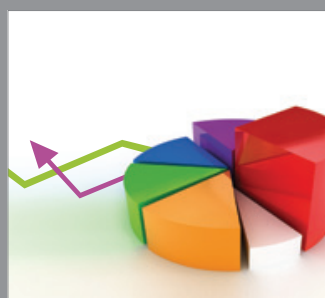

ournal of

Probability and Statistics

Promensencen
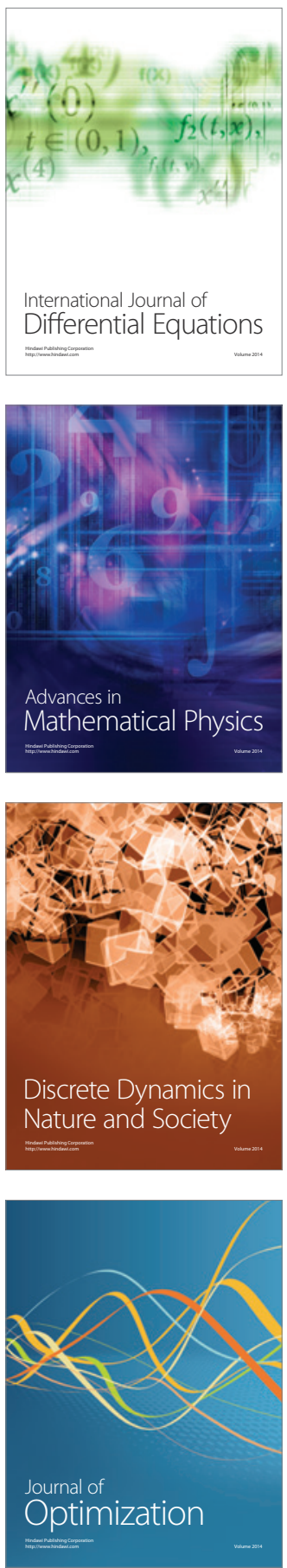